\title{
Ist die Beweglichkeit der HWS bei Patienten mit chronischem Nackenschmerz eingeschränkt?
}

\section{Do Patients with Chronic Neck Pain Demonstrate a Restricted Cervical Range of Motion?}

Autoren

Institut
U. Wolf, S. Mendoza, J. Kiselev, S. Unverzagt, J. Behrens, R. P. Franke

Fachbereich Pflege und Gesundheit, Hochschule Fulda
Schlüsselwörter

- chronischer Schmerz

- Nackenschmerz

- Physiotherapie

- Beweglichkeit

- Validität

Key words

- chronic pain

- neck pain

- physiotherapy

$\checkmark$ mobility

- validity

eingereicht 29.5.2016

akzeptiert 26.3.2017

\section{Bibliografie}

DOI https://doi.org/

10.1055/s-0035-1567213

physioscience 2017 ; 13 :

102-108 (c) Georg Thieme

Verlag KG Stuttgart · New York

ISSN 1860-3092

\section{Korrespondenzadresse}

Prof. Dr. rer. medic. Udo Wolf,

PT

Hochschule Fulda

Fachbereich Pflege und

Gesundheit

Leipziger Str. 12

35037 Fulda

Udo.Wolf@pg.hs-fulda.de

\section{Zusammenfassung}

Hintergrund: Die Untersuchung des Bewegungsumfangs ist fester Bestandteil des Assessments bei Patienten mit Nackenschmerz. Bewegungseinschränkungen gelten als sicheres Zeichen für akute Schmerzzustände. Bei chronischem Nackenschmerz ist das Vorhandensein von Bewegungseinschränkungen jedoch fraglich.

Ziel: Die vorliegende Erhebung untersuchte, ob Patienten mit chronischem Nackenschmerz Bewegungseinschränkungen aufweisen.

Methode: 60 gesunde Probanden und 60 Patienten mit chronischem Nackenschmerz wurden mittels Ultraschalltopometrie während zyklischer Bewegungen der HWS bezüglich des Bewegungsumfangs untersucht und die Mittelwerte beider Gruppen auf signifikante Unterschiede überprüft. Ferner erfolgte die Klassifikation der gemessenen Bewegungsumfänge anhand geschlechts- und altersspezifischer Normwerte als normal, hypermobil oder hypomobil. Für die Validität des Befundes „hypomobil“ in Bezug auf die Erkennung des chronischen Nackenschmerzes wurden die Sensitivität, die Spezifität sowie die positive $(\mathrm{LR}+)$ und die negative Likelihood Ratio (LR-) ermittelt.

Ergebnisse: Die durchschnittlichen Bewegungsumfänge beider Gruppen unterschieden sich in alle Richtungen signifikant. Im Vergleich zu den Normwerten wiesen mehr als drei Viertel der Teilnehmer beider Gruppen eine normale Beweglichkeit auf. Die Sensitivität des Kriteriums „Hypomobilität“ lag bei maximal 0,23, die Spezifität bei über 0,9, die LR+ bei maximal 0,93 und der niedrigste Wert der LR- bei 0,65.

Schlussfolgerung: Beim chronischen Nackenschmerz ist das Bewegungsausmaß der HWS nicht vermindert. Für das Assessment fehlt ein objektiver Parameter, der mit psychosozialen Variablen korreliert und von Physiotherapeuten gemessen werden kann.

\section{Abstract}

Background: Evaluation of the range of motion is a standard procedure in the assessment of patients with neck pain. Restricted movements are considered to be a sure indicator for acute pain. In chronic neck pain the presence of restricted movements is yet doubtful.

Objective: This study examined whether patients with chronic neck pain suffer from restricted movements.

Method: 60 healthy subjects and 60 patients with chronic neck pain were examined during cyclic cervical spinal movements with a view towards range of motion by using ultrasound topometry. The mean amplitude of both groups was analyzed concerning significant differences. In addition, the measured range of motion was classified on the basis of gender and age specific standard values as "normal", "hypermobile" or "hypomobile". Concerning the validity of the diagnosis "hypomobile" in relation to the identification of chronic neck pain sensitivity, specificity and positive (LR+) as well as negative likelihood ratio (LR-) were assessed.

Results: The average of the range of motion in both groups varied significantly for all directions. In comparison to standard values more than three thirds of the participants of both groups showed normal range of motion. The sensitivity of the criterion "hypomobility" attained a maximum of 0.23 , the specificity of more than $0.9, \mathrm{LR}+$ a maximum of 0.93 and the lowest performance of LRwas 0.65

Conclusions: In chronic neck pain the cervical spinal range of motion is not restricted. For the assessment an objective parameter is missing which correlates with psychosocial variables and may be measured by physiotherapists. 


\section{Einleitung}

\section{$\nabla$}

Die Behandlung chronischer Schmerzen unterscheidet sich grundsätzlich von der akuter Schmerzzustände der Wirbelsäule [12, 34]. Ebenso wie im Krankheitsverlauf die Phänomene der zentralen Sensibilisierung gegenüber denen des peripheren nozizeptiven Schmerzes die Oberhand gewinnen, werden aktive Maßnahmen, Training und verhaltenstherapeutische Elemente zunehmend wichtiger. Um die betroffenen Patienten wirksam behandeln zu können, ist es daher bedeutsam, den Übergang von akuten und subakuten zu chronischen Schmerzen sicher zu erkennen. Indizien für diesen Übergang stellen vor allem psychosoziale Variablen dar [28], die sich den behandelnden Physiotherapeuten aufgrund ihrer Spezialisierung auf somatische Zeichen jedoch nicht zwingend offenbaren.

Schmerzen führen in der Regel dazu, dass die betroffenen Personen ihr physiologisches Bewegungsausmaß nicht mehr in vollem Umfang nutzen können. Hier spielen zunächst die Mechanismen der schmerzreflektorischen Hemmung eine Rolle. Persistieren die Beschwerden, so entwickeln sich strukturelle Hypomobilitäten [1]. Auf der Basis dieser Grundannahmen wird das Vorhandensein einer Bewegungseinschränkung als Ausdruck von Schmerzen gedeutet. Umgekehrt gelten Bewegungseinschränkungen als Nachweis für das Vorhandensein von Schmerzen. Die Beweglichkeitsprüfung gehört daher bei Erkrankungen des Bewegungsapparates zum Standardrepertoire des funktionsorientierten Assessments [15]. So empfiehlt auch die zurzeit in Überarbeitung befindliche Leitlinie „Nackenschmerz“ der Deutschen Gesellschaft für Allgemeinmedizin und Familienmedizin die Beweglichkeitsprüfung [6].

Viele klinische Studien stellten Bewegungseinschränkungen bei akutem Nackenschmerz fest. Das Bewegungsausmaß gesunder Probanden unterschied sich signifikant vom Bewegungsausmaß von Patienten mit Nackenschmerzen. Klinische Studien erbrachten die entsprechenden Nachweise durch den statischen Vergleich der Mittelwerte zweier Stichproben [9, 13, 17, 23, 28]. In der klinischen Praxis ist die Beurteilung des Bewegungsausmaßes jedoch in jedem Fall isoliert vorzunehmen. Daher werden die jeweils am Patienten erhobenen Messwerte mit Normwerten verglichen. Hier bieten die von Ryf und Weymann [24] für die Neutral-Null-Methode veröffentlichten Normwerte einen ersten Anhaltspunkt für die Feststellung einer Bewegungseinschränkung oder Überbeweglichkeit.

Die einschlägige Literatur zeigt jedoch, dass das physiologische Bewegungsausmaß vom Alter und Geschlecht der untersuchten Personen abhängt [26, 32]. Deshalb sind der klinischen Beurteilung alters- und geschlechtsspezifische Normwerte zugrunde $\mathrm{zu}$ legen. In gleicher Weise gilt es auch, Behandlungseffekte anhand derartiger Normwerte zu bewerten. Dabei ist zu berücksichtigen, mit welchem Messinstrument die Daten erhoben werden, da hier ebenfalls systembedingte Unterschiede zwischen den absoluten Werten entstehen können.

Als Indikator für das Vorhandensein akuter (und subakuter) Schmerzzustände an der HWS ist das Kriterium „Bewegungseinschränkung“" nachweislich geeignet [9, 13, 17, 23, 28]. Bei persistierenden Beschwerden treten jedoch die objektiven Krankheitszeichen in den Hintergrund. Das Bewegungsausmaß korreliert dann nicht mehr mit dem Schmerz und der Funktionsfähigkeit der HWS [22, 29]. In dieser Phase gewinnen psychosoziale Faktoren zunehmend an Bedeutung [3]. Insbesondere in Fällen, bei denen die geäußerten Beschwerden chronischer Patienten angezweifelt werden, sucht man nach objektiven Krite- rien für das Vorhandensein des Schmerzes. Gleiches gilt auch für die Indikationsstellung für die infrage kommenden physiotherapeutischen Maßnahmen.

Im Rahmen einer großen explorativen Studie wurden daher objektive physikalische Parameter gesucht, die auch im Stadium der Chronifizierung den Schmerz sichtbar machen können. Hierzu erfolgte eine systematische Analyse zahlreicher Parameter. Die vorliegende Arbeit stützt sich auf diesen Datenpool, geht aber ausschließlich der Frage nach, ob Patienten mit chronischem Nackenschmerz noch eine eingeschränkte Beweglichkeit aufweisen (oder ob sich die Beweglichkeit in diesem Stadium wieder normalisiert hat).

\section{Methode \\ $\nabla$ \\ Versuchsteilnehmer, Ein- und Ausschlusskriterien}

Auf der Basis einer Poweranalyse wurde eine Stichprobengröße von 60 gesunden Probanden und 60 Patienten mit chronischem Nackenschmerz ermittelt. In Anlehnung an Kittel et al. [16] galt eine Standardabweichung von 13,6\%, eine Mittelwertdifferenz von $7 \%$ sowie ein Alpha-Fehler von 0,05 und ein Beta-Fehler von 0,2 .

Der Einschluss erfolgte, wenn die angesprochenen Personen mindestens 18 Jahre alt waren und die schriftliche Einwilligung zur Teilnahme an der Studie unterschrieben hatten. Die Teilnehmer beider Gruppen mussten in der Lage und willens sein, die in der Aufklärung beschriebenen und abgebildeten 3 zyklischen Bewegungen je 3-mal durchzuführen. Ausgeschlossen waren Patienten mit Schwindel, deutlich beeinträchtigter Hör- und Sehleistung, Frakturen, rheumatischen, psychischen und neurologischen Erkrankungen sowie Personen, die zum Zweitpunkt der Eingangsuntersuchung einen Schadenersatzprozess wegen Nackenschmerzen führten oder einen noch nicht beschiedenen Rentenantrag gestellt hatten.

Versuchsteilnehmer galten als „gesund“, wenn sie mindestens während der letzten 12 Monate nicht unter Nackenschmerzen gelitten und in den letzten 5 Jahren keine medizinische oder physiotherapeutische Behandlung wegen Nackenschmerzen in Anspruch genommen hatten. Patienten mit „chronischen“ Nackenschmerzen wiesen einen Mindestwert von „1“ für die Schmerzintensität auf einer 11 Punkte umfassenden verbalen Analogskala und anhand des modifizierten Mainz Pain Staging Systems (MPSS; [11]) eine sichere Chronifizierung auf.

Das MPSS ist ein von Gerbershagen und Waisbrod aus der klinischen Praxis heraus entwickelter Fragebogen [11] mit einer von mehreren Autoren nachgewiesenen Konstruktvalidität [10, 14]. Der Fragebogen beurteilt auf 4 Achsen das Schmerzerleben und -verhalten. Auf der 1. Achse werden zeitliche Aspekte des Schmerzerlebens mit 3 Items, auf der 2. Achse räumliche Aspekte des Schmerzes mit 1 Item, auf der 3. Achse das Medikamenteneinnahmeverhalten mit 2 Items und auf der 4 . Achse die Inanspruchnahme medizinscher Leistungen mit 4 Items erfasst.

Für jedes Item können 1-3 Punkte vergeben werden. Durch Addition der Punkte je Achse ergibt sich jeweils ein „Achsenstadium“, aus der Summe der Achsenstadien ein „Gesamtstadium“. Dabei stellt das Gesamtstadium I den niedrigsten, das Gesamtstadium III den höchsten Chronifizierungsgrad dar. Da die Punktwerte zu den insgesamt 10 Items jedoch so angelegt sind, dass auch akute Patienten mit 10 Punkten beurteilt (die Mindestpunktzahl je Item ist 1) und somit dem Gesamtstadium I zugeordnet werden, wurde zusätzlich die 7. Frage aus dem Deutschen 


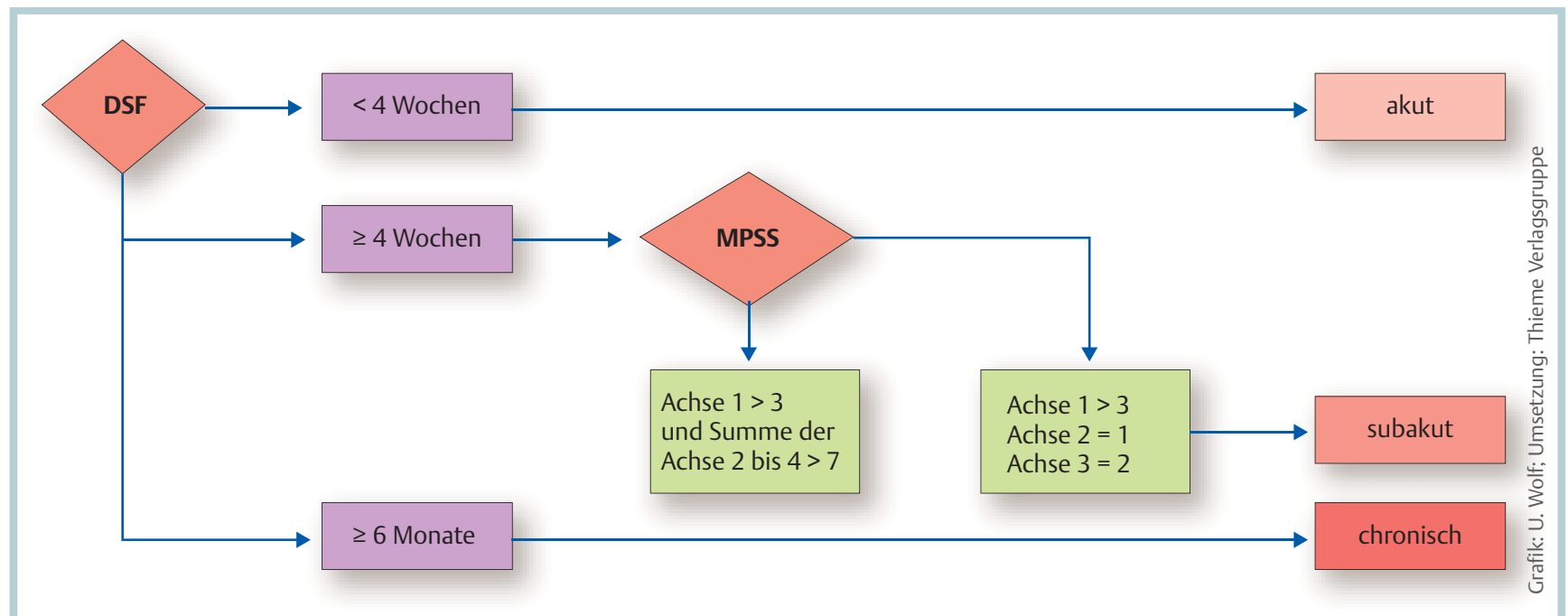

Abb. 1 Algorithmus zum Einschluss als Patient mit chronischen Nackenschmerzen.

Schmerzfragebogen [20] zur Beurteilung der Chronifizierung hinzugenommen. Diese Frage erfasst die Dauer der bestehenden Beschwerden entsprechend der Klassifizierung der International Association for the Study of Pain (IASP). Entsprechend dieser Modifikation galten Patienten als sicher chronisch, wenn sie seit mindestens 6 oder 3 Monaten permanent unter Nackenbeschwerden litten und auf Achse I mehr als 3 Punkte erreichten und die Punktsumme der Achsen II, III und IV größer als 7 war $($ Abb. 1).

\section{Rekrutierung}

Eine wissenschaftliche Mitarbeiterin rekrutierte die Patienten und nahm im Medizinischen Versorgungszentrum „Gesundheitszentrum Marburg“, im neurochirurgischen „Wirbelsäulenzentrum" am Diakoniekrankenhaus Wehrda sowie in der Poliklinik einer orthopädischen Universitätsklinik Marburg den Einschluss vor. Gesunde Probanden wurden aus den Mitarbeitern der Hochschule Fulda, des Fachbereichs Medizin der Philipps-Universität Marburg, des Rheumazentrums Mittelhessen und des Medizinischen Versorgungszentrums „Marburger Gesundheitszentrum“ rekrutiert.

\section{Messung der Beweglichkeit}

Die Beweglichkeit der HWS wurde mittels Ultraschalltopometrie gemessen, indem bei der ausgewählten Applikation „Triple Zervikal“ 3 Schallsender mithilfe eines brillenähnlichen Gestells am Kopf des Patienten angebracht wurden $(\checkmark$ Abb.2). Diese Sender strahlen kontinuierlich in einem Sektor von $120^{\circ}$ Schallwellen mit einer Frequenz von $40 \mathrm{kHz}$ ab. Ein aus 3 piezoelektrischen Ultraschallmikrofonen bestehendes Empfängersystem überträgt die aufgezeichneten Signale zu einem Wandler und von dort an einen konventionellen Computer mit installierter Software des Messsystems. Da zum einen die genaue Position der Sender zueinander definiert und zum anderen die Ausbreitungsgeschwindigkeit von Schall in Luft in Abhängigkeit von der Raumtemperatur bekannt ist, lässt sich anhand der Laufzeit der Signale von einem Sender zu den 3 Empfängern seine jeweilige Entfernung berechnen. Aus der Laufzeitdifferenz eines Signals zu den $3 \mathrm{Mi}$ krofonen können dann die Koordinaten des Senders in den 3 Ebenen bestimmt werden [35].
Die Reliabilität der Bewegungsaufzeichnung wurde für die HWS mehrfach bestätigt $[2,4,5,8,18,19,21,25,30,36$, 37], die Validität an einer kleinen Probandenzahl im Rahmen einer Reliabilitätsstudie [30].

Die Patienten erhielten anhand einer standardisierten Anweisung schriftliche und mündliche die Instruktionen, nach Aufforderungen zunächst 5 zyklische Bewegungen in der Sagittalebene (Flexion und Extension), dann 5 in der Frontalebene (Lateralflexion) und schließlich 5 in der Transversalebene (Rotation) auszuführen. Die Bewegungen sollten endgradig und zügig erfolgen.

\section{Schmerzmessung}

Nach der apparativen Beweglichkeitsmessung wurde die durchschnittliche Schmerzintensität während der insgesamt 30 Bewegungen anhand einer 11 Punkte umfassenden visuellen Analogskala erfasst.

\section{Auswertung}

Zunächst wurde mithilfe der Statistiksoftware SPSS (Statistical Package for the Social Sciences, Version 19.0) anhand des zweiseitigen t-Tests für unabhängige Stichproben die Vergleichbarkeit der Gruppen in Bezug auf das Alter und den Body-Mass-Index (BMI) überprüft. Die ultraschalltopometrisch gewonnenen Daten wurden mithilfe des Programms WinSpine 2.2.3 wie folgt bearbeitet: Zunächst wurde für jede Bewegung der 2.-4. aus den insgesamt 5 Bewegungszyklen herausgeschnitten. Der Grund für dieses Vorgehen war, dass sich in Vorversuchen die „mittleren“ 3 Bewegungszyklen am besten reproduzieren ließen. Das Ausschneiden erfolgte durch manuelles Setzen eines Markers auf der Bewegungskurve für jede Ebene $(\checkmark$ Abb.3). Für die so ermittelten Bewegungsausschläge wurden die Mittelwerte und Standardabweichung für beide Gruppen errechnet und für die Gruppenunterschiede der zweiseitige t-Test für unabhängige Stichproben analysiert.

Zusätzlich wurden die Mittelwerte der maximalen Bewegungsausschläge in die 6 gemessenen Richtungen anhand alters- und geschlechtsspezifischer Normwerte unter Berücksichtigung von 2 Standardabweichungen [4] als bewegungseingeschränkt (hypomobil), normal oder überbeweglich (hypermobil) eingestuft. Unter der Annahme, dass das Vorhandensein einer Hypomobilität chronischen Nackenschmerz andeutet, wurde mittels einer 


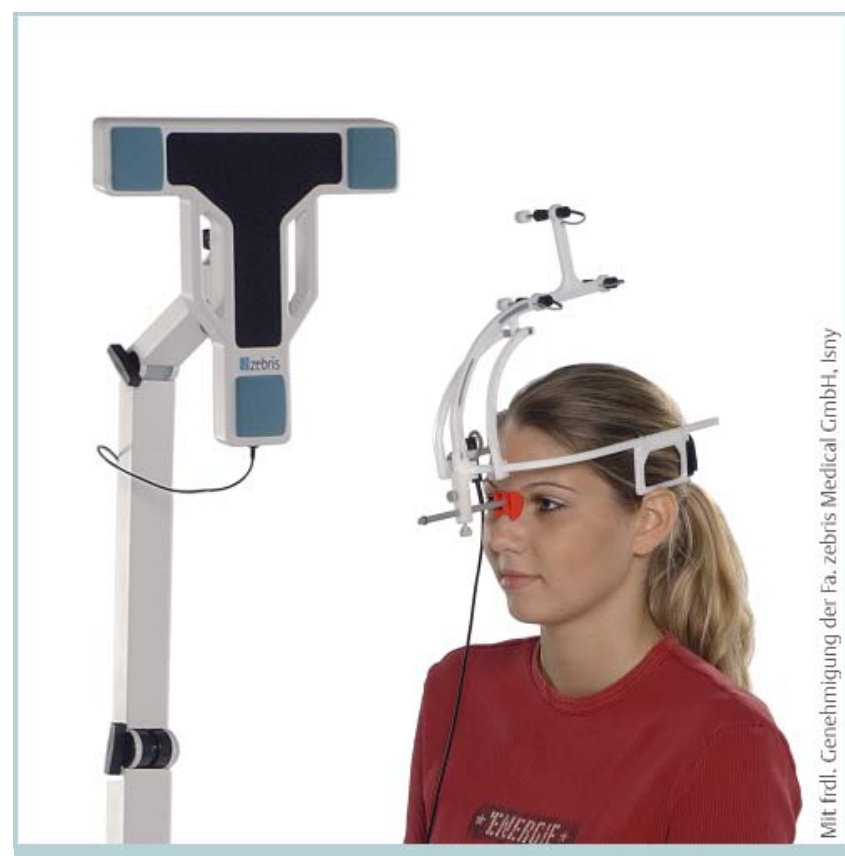

Abb. 2 Ultraschalltopometrische Beweglichkeitsmessung: Senderapplikation (Triple Zervikal).

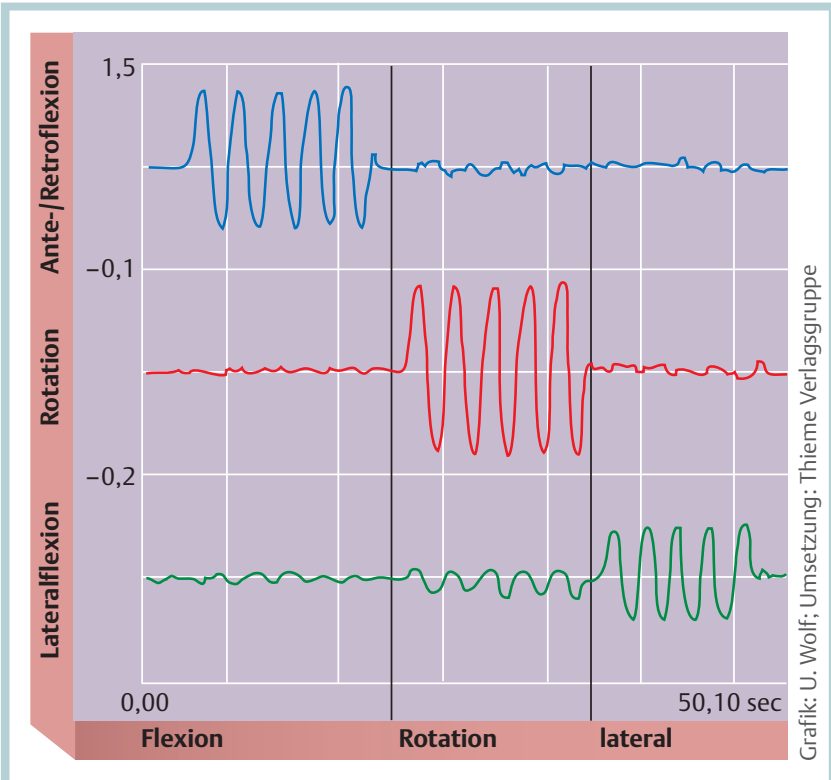

Abb. 3 Bewegungsprotokoll zur Ermittlung der Bewegungsumfänge - hier noch ungeschnitten; Marker (rot) vor dem 1. und hinter dem 5. Zyklus.

4-Felder-Tafel die Sensitivität, Spezifität sowie die positive und die negative Likelihood Ratio ermittelt.

\section{Ethik}

Die zuständige Ethik-Kommission des Fachbereichs Medizin erteilte ein Votum für die vorliegende Studie, die beim Deutschen Register Klinischer Studien DRKS registriert ist.

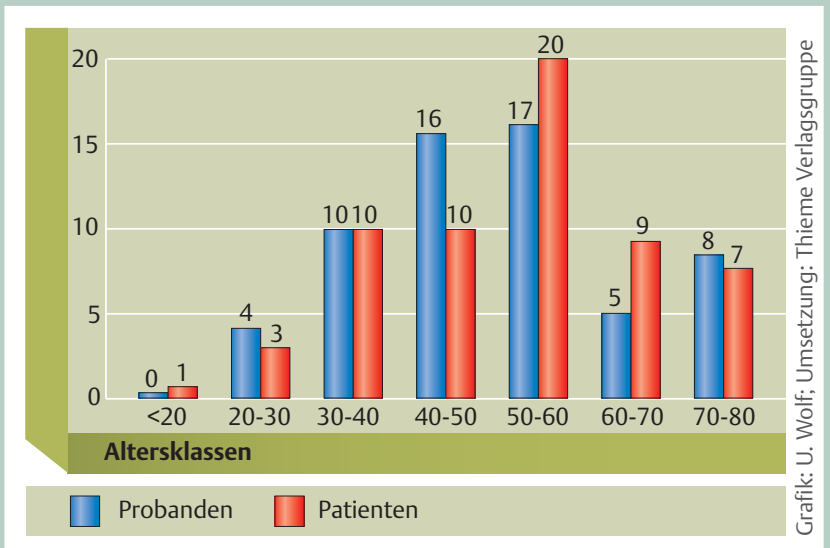

Abb. 4 Verteilung der Patienten und Probanden auf 7 Altersdekaden.

\section{Ergebnisse}

Beschreibung der Stichprobe

Um den erforderlichen Einschluss von 120 Versuchspersonen zu erreichen, wurden 199 Personen befragt. Insgesamt wurden 71 Frauen (32 Probandinnen und 39 Patientinnen) und 49 Männer (28 Probanden und 21 Patienten) eingeschlossen. Somit waren Frauen und Männer in der Kontrollgruppe der Probanden in einem Verhältnis von 32: 28 etwa gleich verteilt, in der Patientengruppe waren jedoch fast doppelt so viele Frauen wie Männer; das Verhältnis betrug 39: 21. In Bezug auf das Alter und den BMI bestanden keine wesentlichen Unterschiede zwischen beiden Gruppen: Die Probanden waren durchschnittlich 50,4 ( $\pm 14,08$ ), die Patienten $52( \pm 13,68)$ Jahre alt. Der BMI der Gesunden lag bei $25,9( \pm 4,81)$, der der Patienten bei 26,2 $( \pm 4,59)$.

Die Anzahl der Teilnehmer innerhalb von 7 Altersdekaden differierte zwischen den Gruppen unterhalb des 40 . Lebensjahres sowie ab dem 70. Lebensjahr um maximal 1 Person, in der Gruppe zwischen 50 und 60 Jahren um 3 Personen. In der Gruppe der 40- bis 50-Jährigen überwogen gesunde Versuchsteilnehmer um 6 Personen. Zwischen dem 60. und 70. Lebensjahr überwogen dagegen kranke Studienteilnehmer um 4 Personen ( $\bullet$ Abb. 4).

\section{Beweglichkeit}

Im Hinblick auf den Bewegungsumfang unterschieden sich Gesunde von Kranken signifikant in allen Bewegungsrichtungen $(\triangleright$ Tab. 1).

Die Bewertung des Bewegungsausmaßes anhand geschlechtsund altersspezifischer Normwerte für die ultraschalltopometrische Bewegungsanalyse an der HWS zeigte, dass über $90 \%$ der gesunden Teilnehmer eine normale Beweglichkeit in Flexion, Extension und Rotation aufwiesen. $80 \%$ der Probanden hatten eine normale Beweglichkeit in Lateralflexion. Bewegungseinschränkungen und vermehrte Beweglichkeiten kamen bei maximal 3\% der Probanden vor. Eine vermehrte Beweglichkeit in Richtung Lateralflexion trat bei $17 \%$ der gesunden Personen auf $(\checkmark$ Abb.5). Bei Patienten mit chronischen Nackenschmerzen fand sich in einem Fall eine vermehrte Beweglichkeit, 10\% (in Richtung Lateralflexion) bis zu $23 \%$ (in Rotationsrichtung) waren vermindert beweglich. Über drei Viertel der untersuchten Patienten wiesen eine normale Beweglichkeit auf (75\% bei Rotation, $80 \%$ bei Flexion und Extension und $88 \%$ bei Lateralflexion). 


\begin{tabular}{|c|c|c|c|c|c|}
\hline \multirow[t]{2}{*}{ Bewegungsumfang } & \multicolumn{2}{|c|}{ gesunde Probanden } & \multicolumn{2}{|c|}{ chronische Patienten } & \multirow{2}{*}{$\begin{array}{l}\text { Vergleich } \\
\text { p }\end{array}$} \\
\hline & MW & STABW & MW & STABW & \\
\hline Flexion & 65,00 & $\pm 13,30$ & 58,85 & $\pm 10,90$ & 0,0065 \\
\hline Extension & 58,02 & $\pm 15,05$ & 44,68 & $\pm 15,25$ & $<0,0001$ \\
\hline Rechtsrotation & 71,64 & $\pm 10,94$ & 60,09 & $\pm 12,08$ & $<0,0001$ \\
\hline Linksrotation & 72,03 & $\pm 11,73$ & 60,85 & $\pm 12,83$ & $<0,0001$ \\
\hline Lateralflexion rechts & 41,65 & $\pm 11,72$ & 33,97 & $\pm 10,27$ & 0,0002 \\
\hline Lateralflexion links & 40,43 & $\pm 14,40$ & 31,49 & $\pm 10,68$ & 0,0002 \\
\hline
\end{tabular}

Tab. 1 Mittelwerte (MW), Standardabweichungen (STABW) und $\mathrm{p}$-Werte $(\mathrm{p})$ von Probanden und Patienten in den untersuchten $\mathrm{Be}$ wegungsrichtungen.

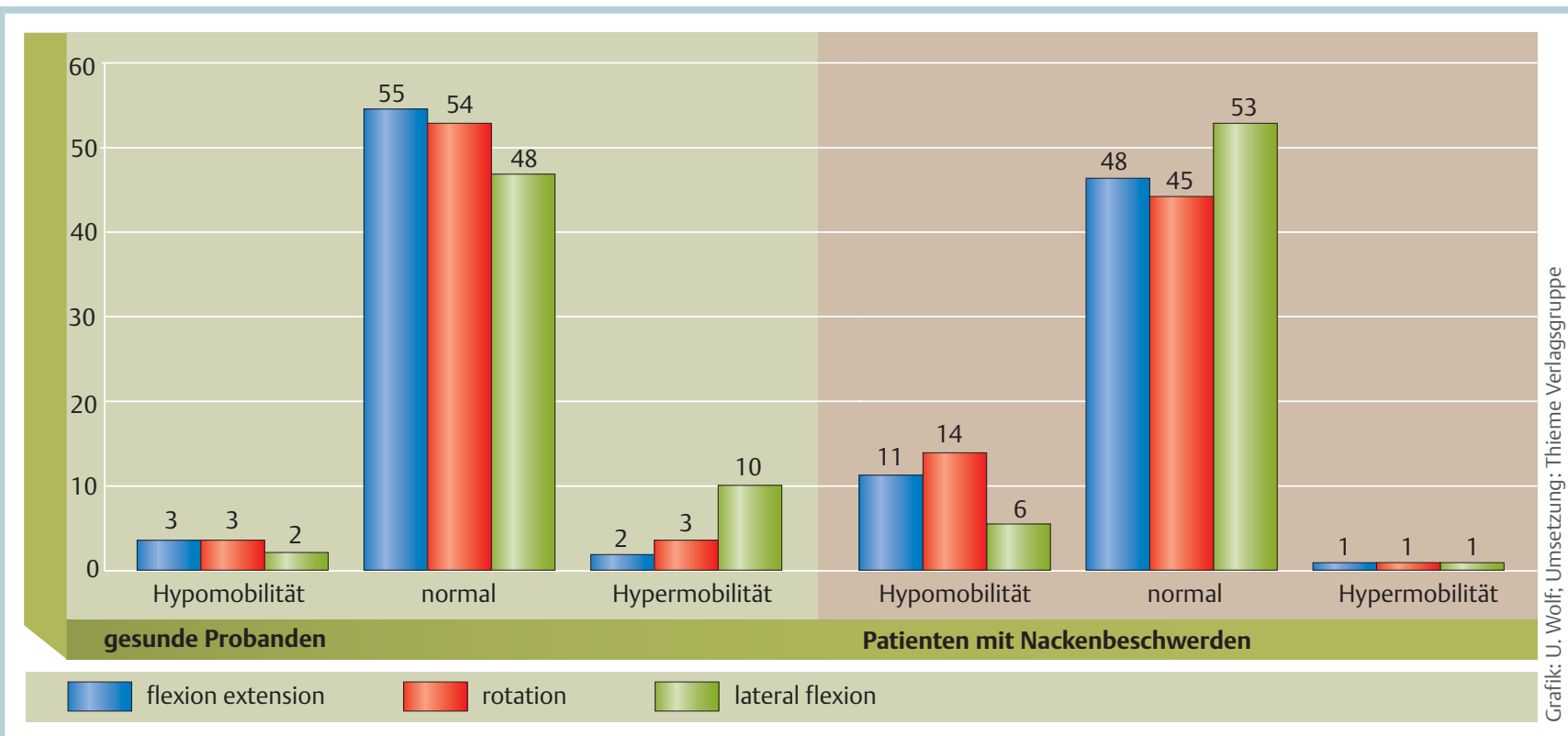

Abb. 5 Häufigkeiten von Hypomobilitäten, Hypermobilitäten und normaler Beweglichkeit bei Probanden und Patienten in den 3 Bewegungsebenen (FlexionExtension, Rotation, Lateralflexion).

Tab. 2 Diagnostische Güte des Kriteriums „Hypomobilität“ für die Diagnose „chronischer Nackenschmerz“.

\begin{tabular}{|c|c|c|c|c|c|c|c|c|}
\hline Hypomobilität & $\begin{array}{l}\text { richtig } \\
\text { positiv }\end{array}$ & $\begin{array}{l}\text { falsch } \\
\text { positiv }\end{array}$ & $\begin{array}{l}\text { falsch } \\
\text { negativ }\end{array}$ & $\begin{array}{l}\text { richtig } \\
\text { negativ }\end{array}$ & SN (KI) & SP (KI) & LR+ & LR- \\
\hline $\begin{array}{l}\text { Flexion und Ex- } \\
\text { tension }\end{array}$ & 11 & 3 & 49 & 57 & $0,18(0,10-0,30)$ & $0,95(0,86-0,99)$ & 0,86 & 0,70 \\
\hline Rotation & 14 & 3 & 46 & 57 & $0,23(0,13-0,36)$ & $0,95(0,86-0,99)$ & 0,81 & 0,65 \\
\hline Lateralflexion & 6 & 2 & 54 & 58 & $0,10(0,04-0,20)$ & $0,97(0,88-1,00)$ & 0,93 & 0,72 \\
\hline
\end{tabular}

KI = Konfidenzintervall; LR+= positive Likelihood Ratio; LR- = negative Likelihood Ratio; SN = Sensitivität; SP= Spezifität.

Setzte man das Vorhandensein einer Hypomobilität als beweisendes Kriterium für chronischen Nackenschmerz an, so ergaben sich die in $\downarrow$ Tab. 2 aufgeführten Werte zur Güte dieses Verfahrens.

Bei entsprechend hoher Spezifität erreichte die Sensitivität je nach Bewegungsrichtung einen Wert von maximal 0,23. Bewegungseinschränkungen kamen also bei weniger als einem Viertel der kranken Testpersonen vor (richtig-positive Tests). Die positive Likelihood Ratio lag für alle Bewegungsrichtungen unter 1. Somit ist die Wahrscheinlichkeit, dass Testpersonen mit eingeschränkter Beweglichkeit unter chronischen Nackenschmerz leiden, nicht erhöht. Die negative Likelihood Ratio betrug für alle Bewegungsrichtungen über 0,6. Die Wahrscheinlichkeit mit nor- maler Beweglichkeit unter chronischem Nackenschmerz zu leiden, war damit auf weniger als die Hälfte reduziert.

\section{Diskussion}

Die vorliegenden Ergebnisse zeigen, dass sich die Mittelwerte der Beweglichkeit der Patienten mit chronischem Nackenschmerz signifikant von denen der Kontrollgruppe mit gesunden Personen unterscheiden. Die anhand alters- und geschlechtsspezifischer Normwerte als normale, verminderte oder vermehrte Beweglichkeit bewerteten Bewegungsumfänge jedes einzelnen Patienten verdeutlichen jedoch, dass mehr als drei Viertel der Pa- 
tienten mit chronischem Nackenschmerz keine Bewegungseinschränkung aufwiesen.

Ein ähnlich kritisches Bild in Bezug auf die Eignung des Kriteriums „Bewegungsausmaß“ zeichnete auch Yliemen [38], in dessen Studie beim chronischen Nackenschmerz die Beweglichkeit ebenfalls nicht mit der Beschwerdesymptomatik der Patienten korrelierte.

Andere Autoren bewerteten die Relevanz der Beweglichkeitsprüfung positiv und konnten eine veränderte Beweglichkeit bei Patienten mit chronischem Nackenschmerz nachweisen [23, 28]. Die beschriebenen Unterschiede beziehen sich allerdings auf statistische Mittelwertvergleiche zweier Gruppen. Eine Bewertung anhand von alters- und geschlechtsspezifischen Normwerten nahmen diese Arbeiten nicht vor [23, 28].

Als Erklärung für die unterschiedlichen Ergebnisse kann die starke interindividuelle Variabilität des normalen Bewegungsausmaßes herangezogen werden. Dies dokumentieren eindrucksvoll die von Castro et al. [4] veröffentlichten Normwerte für die Beweglichkeitsmessung an der HWS: Die Standardabweichungen reichen von $10,0^{\circ}$ bei Männern im Alter zwischen 50 und 59 Jahren bis zu $31,8^{\circ}$ bei Frauen zwischen 30 und 39 Jahren [4]. Die statistischen Vergleiche von Gruppenmittelwerten dürften daher stark von der Wahl der Stichprobe abhängen.

Die große Variabilität der normalen Beweglichkeit der HWS hat vielfältige Ursachen. So kann die Beweglichkeit des Nackens z. B. durch die Haltung $[7,31]$ und die Bewegungskoordination zwischen der HWS und den oberen thorakalen Segmenten [33] beeinflusst werden. Weiteren Einfluss auf das Bewegungsausmaß können auch sportliche Beanspruchungen haben [37]. Dies erklärt einerseits die unterschiedlichen Studienergebnisse und bestätigt andererseits die Ergebnisse dieser Studie dahingehend, dass das Bewegungsausmaß zu vielen Einflussgrößen unterworfen ist, als dass es zur Identifizierung von Patienten mit chronischem Nackenschmerz geeignet sein könnte.

\section{Schlussfolgerungen \\ $\nabla$}

Das Bewegungsausmaß spielt - anders als beim akuten und subakuten Nackenschmerz - beim chronischen Nackenschmerz eine untergeordnete Rolle und ist daher zurückhaltend zu interpretieren. Die klassische Diagnostik ist daher überwiegend auf die Validität von Patientenangaben angewiesen. Es fehlt ein objektives Kriterium zur Diagnostik chronischer Nackenschmerzen. Idealerweise wird künftig ein objektiver Parameter identifiziert, der mit den subjektiven Parametern korreliert, mit Methoden der Physiotherapie erhoben werden kann und die Gütekriterien für Tests erfüllt.

Interessenkonflikt: Die Autoren geben an, dass kein Interessenkonflikt besteht.

\section{Literatur}

1 Van den Berg F. Angewandte Physiologie. Bd. 1: Das Bindegewebe des Bewegungsapparates verstehen und beeinflussen. Stuttgart: Thieme; 2011

2 Cagnie B. Cools A. De Loose V et al. Reliability and normative database of the Zebris cervical range-of-motion system in healthy controls with preliminary validation in a group with neck pain. J Manipulative Physiol Ther 2007; 30: $450-455$

3 Carroll LJ. Hogg-Johnson S. van der Velde G et al. Course and prognostic factors for neck pain in the general population: results of the Bone and Joint Decade 2000-2010 Task Force on Neck Pain and Its Associated Disorders. Spine 2008; 33: 75 -82
4 Castro W. Sautmann A. Schilgen M et al. Noninvasive Three-Dimensional Analysis of Cervical Spine Motion in Normal Subjects in Relation to Age and Sex. Spine 2000; 25: $443-449$

5 Demaille-Wlodyka S. Chiquet C. Lavaste JF et al. Cervical range of motion and cephalic kinesthesis. Spine 2007; 32: E254-E261

6 Deutsche Gesellschaft für Allgemeinmedizin und Familienmedizin. Leitlinie Nr. 13 Nackenschmerzen - Langfassung. http://www.awmf. org/uploads/tx_szleitlinien/053-0071_S1_Nackenschmerz_2017-01. pdf (29.05.2016)

7 Dunleavy K. Goldberg A. Comparison of cervical range of motion in two seated postural conditions in adults 50 or older with cervical pain. J Man Manip Ther 2013; 21: 33-39

8 Dvir Z. Prushansky T. Reproducibility and instrument validity of a new ultrasonography-based system for measuring cervical spine kinematics. Clinl Biomech 2000; 15: 658 - 664

9 Fletcher JP. Bandy WD. Intrarater reliability of CROM measurement of cervical spine active range of motion in persons with and without neck pain. J Orthop Sports Phys Ther 2008; 38: 640-645

10 Frettlöh J. Maier C. Gockel H et al. Validation of the German Mainz Pain Staging System in different pain syndromes. Schmerz 2003; 17: 240 251

11 Gerbershagen HU. Waisbrod $H$. Chronic pain management. Part I: Factors involved in comprehensive pain patient care evaluation. Schmerz 1986; 2: 55-59

12 Gross A. Kay TM. Paquin JP. Cervical Overview Group. et al. Exercises for mechanical neck disorders. Cochrane Database Syst Rev 2015; 28: 1

13 Guo LY. Lee SY. Lin CF et al. Three-dimensional characteristics of neck movements in subjects with mechanical neck disorder. J Back Musculoskelet Rehabil 2012; 25: 47-53

14 Hampel P. Moergel MF. Staging of pain in patients with chronic low back pain in inpatient rehabilitation: validity of the Mainz Pain Staging System of pain chronification. Schmerz 2009; 23: 154-165

15 Harris N. Ali F. Miller MD. Examination Techniques in Orthopaedics. Cambridge: Cambridge University Press; 2014

16 Kittel R. Bittmann F. Badtke G et al. Objektivierte Beurteilung der aktiven zyklischen Halswirbelsäulenrotation bei Funktionsstörungen. Man Med 2002; 40: 262-266

17 Lee H. Nicholson LL. Adams RD. Cervical range of motion associations with subclinical neck pain. Spine 2004; 29: 33-40

18 Malmström EM. Karlberg M. Melander A et al. Zebris versus Myrin. A comparative study between a three-dimensional movement analysis and an inclinometer/compass method. Spine 2003; 28: 433 -440

19 Mannion AF. Klein GN. Dvorak J et al. Range of global motion of the cervical spine: intra-individual reliability and the influence of measurement device. Eur Spine J 2000; 9: 379-385

20 Nagel B. Gerbershagen HU. Entwicklung und empirische Überprüfung des Deutschen Schmerzfragebogens der DGSS. Schmerz 2002; 16: $263-270$

21 Natalis M. König A. Nichtinvasive, akkurate und reliable Messung der Halswirbelsäulenbeweglichkeit mittels ultraschallgestützter 3D-Echtzeit-Bewegungsanalyse. Ultraschall in Med 1999; 20: 70-73

22 Rodriquez AA. Burns SP. Assessment of chronic neck pain and a brief trial of cervical strengthening. Am J Phys Med Rehabil 2008; 87: 903-909

23 Rudolfsson T. Björklund M. Djupsjöbacka M. Range of motion in the upper and lower cervical spine in people with chronic neck pain. Man Ther 2012; 17: 53 - 59

24 Ryf C. Weymann A. Range of Motion - AO Neutral-0 Method. Measurement and Documentation. New York: Thieme; 1999

25 Schreiber TU. Smolenski UC. Seidel EJ. Dreidimensionale Bewegungsanalyse zur Funktionsbeurteilung der Halswirbelsäule - Messverfahren und Reliabilität. Phys Rehab Kur Med 2001; 11: 113-122

26 Sforza C. Grassi G. Fragnito N et al. Three-dimensional analysis of active head and cervical spine range of motion: effect of age in healthy male subjects. Clin Biomech Bristol Avon 2002; 17: 611 -614

27 Shahidi B. Curran-Everett D. Maluf KS. Psychosocial, Physical, and Neurophysiological Risk Factors for Chronic Neck Pain: A Prospective Inception Cohort Study. J Pain 2015; 16: 1288 - 1299

28 Shahidi B. Johnson CL. Curran-Everett D et al. Reliability and group differences in quantitative cervicothoracic measures among individuals with and without chronic neck pain. BMC Musculoskelet Disord 2012; 13: 215

29 Sjölander P. Michaelson P. Jaric S et al. Sensimotor disturbances in chronic neck pain - range of motion, peak velocity, smoothness of motion and repositioning acuity. Man Ther 2008; 13: $122-131$ 
30 Strimpakos N. Sakellari V. Gioftsos G et al. Cervical spine ROM measurements: optimizing the testing protocol by using a 3D ultrasound-based motion analysis system. Cephalgia 2005; 25: 1133 -1145

31 Takasaki H. Hall T. Oshiro S et al. Normal kinematics of the upper cervical spine during the Flexion-Rotation Test - In vivo measurements using magnetic resonance imaging. Man Ther 2011; 16: 167-171

32 Tommasi DG. Foppiani AC. Galante D et al. Active head and cervical range of motion: effect of age in healthy females. Spine 2009; 34 : $1910-1916$

33 Tsang SMH. Szeto GPY. Lee RYW. Normal kinematics of the neck: the interplay between the cervical and thoracic spines. Man Ther 2013; 18: $431-437$
34 Vincent K. Maigne JY. Fischhoff C et al. Systematic review of manual therapies for nonspecific neck pain. Joint Bone Spine 2013; 80: 508-515

35 Vogt L. Banzer W. Reproduzierbarkeit thorakaler und lumbaler Wirbelsäulenbewegungen mit der 3D Ultraschalltopometrie. Phys Rehab Kur Med 1997; 7: $21-25$

36 Wainner R. Fritz JM. Irrgang JJ et al. Reliability and Diagnostic Accuracy of the Clinical Examination and Patient Self-Report Measures for Cervical Radiculopathy. Spine 2003; 28: 52-62

37 Wang SF. Teng CC. Lin KH. Measurement of cervical range of motion pattern during cyclic neck movement by an ultrasound-based motion system. Man Ther 2005; 10: 68 - 72

38 Ylinen J. Physical exercises and functional rehabilitation for the management of chronic neck pain. Eura Medicophys 2007; 45: 119-122 